\title{
VIOLENCIA ENTRE PAREJA ÍNTIMA EN CONTRA DE LA MUJER: UN ANÁLISIS DE EMPODERAMIENTO A TRAVÉS DE LA EDUCACIÓN
}

\author{
Diana M. Cavazos \\ Universidad Internacional Iberoamericana (México)
}

\begin{abstract}
Resumen. El marco teórico de empoderamiento fue utilizado para exponer la relevancia del empoderamiento de mujeres víctimas de violencia entre pareja íntima, a través de programas educativos, para lograr su independencia alejadas de compañeros íntimos violentos. Metodología: Este reporte es un análisis retrospectivo, cuantitativo, no experimental, de datos que exploran los resultados de la toma de decisiones de las mujeres abusadas, después de haber participado en programas educativos de empoderamiento. La muestra incluyó 2837 expedientes de mujeres adultas, víctimas de diferentes tipos de abuso causado por su pareja íntima. Los expedientes fueron clasificados según la inscripción en los módulos educativos ofrecidos en el albergue. Los datos recopilados fueron analizados utilizando medidas estadísticas descriptivas para determinar la frecuencia marginal de dos resultados binarios. Resultados: El nivel de participación en los programas educativos fue extremadamente bajo; aun así, las mujeres abusadas que son empoderadas para obtener alojamiento seguro pudieron alejarse de su pareja violenta; sin embargo, aun siendo empoderadas para conseguir empleo o con las destrezas para manejar sus finanzas, la mayoría de la mujeres no obtuvieron empleo ni los recursos económicos para retirarse de la relación violenta. Discusión: Se necesitan programas efectivos que empoderen a la mujer a retirarse del ciclo de violencia entre pareja íntima. Los esfuerzos de investigación son esenciales para determinar la efectividad de programas educativos que empoderan a la mujer a ser participantes activas en su jornada de recuperación de violencia entre pareja íntima.
\end{abstract}

Palabras clave: Violencia entre pareja íntima, empoderamiento, empoderamiento económico, empleo, alojamiento.

\section{INTIMATE PARTNER VIOLENCE AGAINST WOMEN: AN ANALYSIS OF EMPOWERMENT THROUGH EDUCATION}

\begin{abstract}
Introduction: The empowerment model was used as a framework to expose the relevance of empowerment of women victims of intimate partner violence, through educational programs, to achieve independence away from violent intimate partners. Methodology: This report is a retrospective, quantitative, non-experimental analysis of data that explored abused women's decision making outcomes after participating in empowering educational programs offered at a residential battered women's shelter. The sample included 2837 records of adult sheltered women who had reported one or more types of abuse from an intimate partner. Records were sub-grouped according to women's enrollment in educational modules offered at the selected residential shelter. The data collected was analyzed using descriptive statistical analysis of measures to determine the marginal frequencies of two binary outcomes. Results: Although a poor level of participation in the shelter's educational programs was observed, the results included positive and negative associations in the different hypothesis. Battered women who are empowered to obtain safe housing are able to move away from their abusive partner; nevertheless, despite being empowered to pursue employment or being empowered with financial management skills, most battered women did not attain an occupation nor the financial resources to break away from an intimate partner violent relationship. Discussion: Effective programs are needed to empower women to break away from the cycle of intimate partner violence. Research efforts are essential to determine the
\end{abstract}


effectiveness of educational programs that empower victims of intimate partner violence to be active participants in their road to recovery from intimate partner violence.

Keywords: Intimate partner violence, empowerment, economic empowerment, employment, housing.

\section{VIOLENCIA DOMESTICA CONTRA MULHERES: UMA ANALISE DO FORTALECIMENTO ATRAVÉS DA EDUCAÇÃO}

Resumo. Introdução: O modelo de fortalecimento foi utilizado como estrutura para expor a relevância do empoderamento das mulheres vítimas de violência domestica, por meio de programas educacionais, para preparar-las a serem independentes sem seus parceiros Metodologia: Este relatório é uma análise retrospectiva, quantitativa, não-experimental de dados baseados nas decisões tomadas pelas mulheres abusadas, depois de participar nos programas educacionais de capacitação oferecidos em um abrigo para mulheres maltratadas. $\mathrm{O}$ estudo incluiu 2837 registros de mulheres protegidas que relataram um ou mais tipos de abuso de um parceiro íntimo. Os resultados foram subgrupados de acordo com o programa educacional selecionado por cada mulher. Os dados coletados foram analisados utilizando análise estatística descritiva de medidas para determinar as frequências marginais de dois resultados. Resultados: Embora tenha sido observado pouca participação nos programas educacionais do abrigo, os resultados incluíram associações positivas e negativas nas diferentes hipóteses. As mulheres maltratadas que foram capacitadas para obter habitação segura se tornaram capazes de se afastar de seu parceiro abusivo; no entanto, apesar de terem sido capacitadas a trabalhar ou habilitadas para organizar suas finanças, a maioria das mulheres maltratadas não conseguiram emprego nem os recursos financeiros para se afastarem do relacionamento violento. Discussão: São necessários programas eficazes para capacitar as mulheres a se afastarem do ciclo da violência domestica. Pesquisas são essenciais para determinar a eficácia dos programas educacionais que capacitam as vítimas da violência domestica a serem participantes ativos em seu caminho na recuperação da violência domestica.

Palavras-chave: Violência domestica, fortalecimento, empoderamento econômico, emprego, habitação.

\section{Introducción}

La violencia entre pareja íntima, antes conocida como violencia doméstica, continua siendo un problema social complejo alrededor del mundo. La violencia entre pareja íntima en contra de la mujer se ha evidenciado a través de varios periodos históricos en la mayoría de las sociedades, lo cual establece precedentes que apoyan transparentemente este tipo de violencia (Criminal Justice, n. d.).

Antiguas civilizaciones con culturas patriarcales reforzaron la subyugación de la mujer ante el hombre y a pesar de que la historia de la civilización indica que la sociedad ha evolucionado y que la tendencia patriarcal ha perdido gran parte de su poder a causa de movimientos sociales que benefician a la mujer, el fenómeno de la violencia entre pareja íntima en contra de la mujer aún existe (Criminal Justice, n. d.). Los estudios sobre la violencia entre pareja íntima en contra de la mujer se han propagado significativamente en la última década, haciendo posible la identificación de la trascendencia de este problema; sin embargo, investigadores en alrededor del mundo continúan evaluando los factores que determinan cuando una mujer se siente empoderada para reportar el abuso en contra de ella y abandonar una relación íntima violenta (Wihbey, 2015). 
La decisión de estudiar la participación de mujeres en módulos educativos de empoderamiento fue tomada teniendo en consideración que la sociedad erróneamente afirma que la mujer abusada puede abandonar una relación violenta si en realidad quisiera hacerlo; sin embargo, la sociedad también pasa por alto las barreras circunstanciales que evitan que una mujer pueda abandonar una relación abusiva. Considerando los problemas específicos que generalmente influyen en las decisiones de cada individuo para poder abandonar una relación violenta, las siguientes hipótesis fueron desarrolladas:

H1i: Las mujeres abusadas que tienen acceso a programas educativos que las empoderan con las destrezas y recursos para obtener alojamiento seguro está positivamente asociado con el hecho de abandonar una relación de pareja intima violenta y la obtención de alojamiento seguro lejos del abusador.

H10: Las mujeres abusadas que tienen acceso a programas educativos que las empoderan con las destrezas y recursos para obtener alojamiento seguro no está positivamente asociado con el hecho de abandonar una relación de pareja intima violenta y la obtención de alojamiento seguro lejos del abusador.

H2i: Las mujeres abusadas que tienen acceso a programas educativos que las empoderan con las destrezas y recursos para manejar sus finanzas está positivamente asociado con el hecho de incrementar su ingreso económico, lejos del abusador.

H20: Las mujeres abusadas que tienen acceso a programas educativos que las empoderan con las destrezas y recursos para manejar sus finanzas no está positivamente asociado con el hecho de incrementar su ingreso económico, lejos del abusador.

$\mathrm{H} 3 \mathrm{i}$ : Las mujeres abusadas que tienen acceso a programas educativos que las empoderan con las destrezas y recursos para obtener empleo está positivamente asociado con el hecho de unirse a las fuerzas laborales, lejos del abusador.

H30: Las mujeres abusadas que tienen acceso a programas educativos que las empoderan con las destrezas y recursos para obtener empleo no está positivamente asociado con el hecho de unirse a las fuerzas laborales, lejos del abusador.

La sociedad continúa especulando que las tácticas existentes implementadas para ayudar a las mujeres abusadas lograrán reducir drásticamente la incidencia de violencia entre pareja íntima, ignorando los defectos en las estrategias utilizadas actualmente, las cuales han demostrado ser mayormente infectivas y en ocasiones perjudiciales. Las estrategias existentes revelan falacias y conceptos erróneos significativos sobre la naturaleza de la violencia entre pareja intima, sobre su incesante incidencia, la variedad de medidas diseñadas para erradicarla, y sobre la necesidad de sobrellevar y fomentar este comportamiento violento, derivado de las raíces profundas que apoyan las normas culturales de sociedades pretéritas (Aiken \& Goldwasser, 2010).

El objetivo principal de este estudio retrospectivo es evaluar los resultados de empoderamiento a través de módulos educativos ofrecidos a mujeres víctimas de violencia entre pareja intima, residentes en un albergue para mujeres maltratadas. El promedio nacional de incidencia de violencia entre pareja íntima ha disminuido, sin embargo, el estado de Texas figura en segundo lugar en términos de llamadas telefónicas a la línea de emergencia para víctimas de violencia, ubicando a la ciudad de San Antonio en 4to lugar de donde estas llamadas se originan (Texas Council on Family Violence, 2014); por esta razón, los datos archivados en un albergue para mujeres maltratadas, en la ciudad de San Antonio, Texas, fueron analizados para este estudio. 
Las creencias tradicionales patriarcales que estipulan que el hombre tiene el derecho de controlar a la mujer crea en la mujer vulnerabilidad a la violencia física, emocional, y sexual a manos del hombre, quebrantando seriamente su bienestar físico, mental y social (World Health Organization, 2009). La incidencia histórica de la violencia basada en género, en contra de la mujer, sugiere que este tipo de violencia no es una ocurrencia casual si no una manifestación de una cultura patriarcal incrustada sistemáticamente en sociedades pasadas y presentes. La teoría del patriarcado se fomenta en círculos privados y públicos, validada vigorosamente por grupos e individuos que la apoyan abierta y clandestinamente, delineando y reforzando normas restrictivas basadas en género a favor del hombre. Las creencias culturales de cómo debe ser tratada una mujer, frecuentemente justifican la desigualdad de género y la violencia en contra de ella (Asian Pacific Institute on Gender-Based Violence, 2015).

El concepto de la violencia entre pareja intima en contra de la mujer surgió en los Estados Unidos como un gran problema social en la década de los 70's, pero no fue hasta finales del siglo XX que la violencia entre pareja íntima fue definida como un crimen, lo cual justificó la intervención del sistema judicial penal para afrontar su incidencia (Dobash \& Dobash, 1979, citado en Erez, 2002). Los investigadores sociales continúan identificando elementos que empoderan a las mujeres víctimas de violencia entre pareja íntima para reportar el abuso en contra de ellas y abandonar su relación violenta (Wihbey, 2015). Para los investigadores es de gran importancia poder determinar los hechos que fomentan en las víctimas de violencia entre pareja intima una actitud de empoderamiento para abandonar una relación violenta, en contraste con la víctimas que deciden quedarse con su abusador (Temple, Le, Muir, Goforth, \& McElhany, 2013).

Kreager, Felson, Warner, and Wenger (2013) establecieron en su estudio titulado La educación de la mujer, violencia marital y divorcio: una perspectiva de intercambio social, que tradicionalmente, cuando las mujeres son empoderadas con suficientes recursos, dependen menos del hombre y tienen más oportunidades fuera de una relación, teniendo también más oportunidades para abandonar una relación de pareja íntima abusiva. Los resultados de otras investigaciones también revelan que la educación y los recursos económicos ayudan a la mujer a incrementar la estabilidad en una relación entre pareja intima no abusiva y promueve la disolución de relaciones entre pareja intima abusivas (Dare, Guadagno, \& Muscanell, 2013).

Las raíces de la violencia entre pareja intima se atribuyen a una gran variedad de factores culturales, sociales, económicos, y psicológicos. Empoderar a la mujer instando comportamientos que promueven la búsqueda de ayuda y empoderarla con el acceso a recursos disponibles, es un elemento fundamental para asegurar que las acciones tomadas para abandonar una relación violenta sean efectivas. Involucrarse en una relación de violencia entre pareja íntima no siempre puede ser prevenible; sin embargo, abandonar una relación violenta si lo es, aunque es un proceso de factores complejos que deben ser evaluados por la víctima para establecer su seguridad y bienestar y la de sus hijos. Aún con múltiples barreras para romper el ciclo de violencia, muchas mujeres logran abandonar sin peligro su relación violenta de pareja intima (Missouri Coalition Against Domestic and Sexual Violence, 2014).

El marco teórico utilizado para guiar este estudio fue el marco teórico de empoderamiento de la mujer. El "empoderamiento" ha sido definido en términos generales como el proceso de poder influenciar eventos y resultados de importancia para un individuo o grupo; se conoce como un modelo dinámico con diferente 
significado para cada persona, cambiando constantemente según el tiempo y circunstancias (Vaile-Wright, Perez, \& Johnson, 2012). Más allá de tratar de erradicar la violencia, la cual no es controlada por la víctima, el enfoque del empoderamiento utiliza la dispersión de conocimientos, educación, y psicoterapia para crear un conjunto de servicios que las víctimas pueden controlar (Ofstehage, Gandhi, Sholk, Radday, \& Stanzler, 2011).

El objetivo principal de este estudio retrospectivo fue evaluar los resultados de empoderamiento a través de módulos educativos ofrecidos a las mujeres víctimas de violencia entre pareja íntima, residentes en un albergue de mujeres maltratadas. Específicamente, este estudio evaluó la incidencia de participación de las mujeres albergadas, en programas informativos ofrecidos en el albergue. Adicionalmente, este estudio midió la incidencia de obtención de alojamiento, la incidencia de logros financieros y la incidencia de logros laborales de las mujeres albergadas que participaron en sesiones informativas sobre obtención de alojamiento seguro, manejo de finanzas y destrezas para obtener empleo, al salir del albergue.

Incluye la presentación del documento y el análisis de la literatura sobre el tema, con especial énfasis en las investigaciones previas que justifican el estudio y que luego se contrastarán en la discusión de los resultados.

\section{Método}

Este estudio es un análisis retrospectivo, cuantitativo, no-experimental, el cual examinó los datos recopilados en un transcurso de 5 años en un albergue para mujeres maltratadas en la ciudad de San Antonio, Texas, en los Estados Unidos. Este estudio examinó la relación entre cursos didácticos específicos ofrecidos a las mujeres residentes del albergue y los efectos secundarios que estos módulos instruccionales tuvieron en las mujeres participantes para sentirse empoderadas con los recursos económicos, un hogar seguro, o un empleo adecuado que le provea ingresos económicos, para apoyar su decisión de salir de su relación violenta entre pareja íntima y vivir alejada e independientemente de su abusador a su salida del albergue.

La población seleccionada consistió de mujeres adultas, de 18 años de edad o mayores, quienes reportaron haber experimentado violencia de cualquier tipo ente pareja intima, y quienes residieron en al albergue de mujeres maltratadas entre el año 2010 y 2015, en donde participaron en los módulos educativos Empoderamiento Económico, Preparación para el Trabajo, o Abriendo la Cerradura de Casa. En total, se utilizaron 2837 expedientes de los archivos del albergue, los cuales proveyeron información sobre las mujeres que buscaron refugio a causa de la violencia entre pareja íntima.

Los datos utilizados fueron recopilados y archivados por el personal del albergue con el programa de recopilación de datos "ALICE Versión 8.0", fiscalizado por el personal administrativo; los datos pertinentes fueron reunidos y la información sobre las variables establecidas fue codificada y medida de acuerdo con todas las reglas y protocolos característicos de un estudio de investigación retrospectivo.

Los datos recopilados fueron analizados usando análisis de medidas estadísticas para determinar la frecuencia marginal de dos resultados binarios. En este estudio, la prueba no-paramétrica (sin-distribución) McNemar fue utilizada para evaluar si hubo un cambio en proporciones estadísticas significativas en un rasgo dicotómico en dos puntos 
de tiempo en la misma población. Adicionalmente, se hizo un análisis descriptivo para determinar las medidas de tendencia central en los indicadores sustractivos.

\section{Resultados}

Los resultados proporcionaron información significativa sobre el empoderamiento a través de la educación, comparando específicamente las variables de alojamiento, ingreso económico y empleo. Los resultados del análisis sociodemográfico fueron consistentes con los resultados de estudios previos. La edad de las victimas osciló entre los 18 y 31 años de edad, con una edad media de 32 años y con el porcentaje más alto en el grupo de 18 y 44 años de edad. Con respecto al estado civil, los porcentajes más altos incluyeron a las mujeres solteras, seguido por mujeres casadas y las mujeres separadas después de éstas.

Un alto porcentaje (44.4\%) fue representado por las viviendas ocupadas por una sola persona. Mientras $32.2 \%$ de las participantes vivían con amistades o familiares, $44.8 \%$ rentaban su casa, $11.2 \%$ reportaron ser propietarias de su casa y $4.9 \%$ vivían en albergues para personas sin hogar. Solamente $0.8 \%$ vivían en viviendas subvencionadas y $0.3 \%$ vivan en viviendas de transición. Aunque no hubo especificaciones disponibles, 4.4\% de las participantes reportaron tener otro tipo de alojamiento. Faltaron los datos de $1.4 \%$ de los casos. El número de estadías registradas en albergue por participante osciló entre 1 y 9. La mayoría de las participantes $(83.9 \%)$ tuvo una sola estadía durante el periodo del estudio y $11.6 \%$ tuvo dos o más estadías. $4.1 \%$ tuvo tres a cuatro estadías y $.4 \%$ tuvo cinco o más.

Casi 93\% de las participantes sufrieron más de un tipo de violencia. El tipo de abuso reportado más frecuentemente fue el abuso verbal-emocional-psicológico (95.7\%), seguido por abuso físico (85.4\%). Más de la mitad de las mujeres participantes reportaron haber experimentado abuso relacionado con la destrucción de su propiedad (58.0\%), asfixia/estrangulación (53\%) o confinamiento (50.2\%). El abuso sexual fue reportado por $23.9 \%$ de las mujeres, $12.6 \%$ reportó el abuso a sus mascotas, $43.4 \%$ reportó haber sido acechadas y $37.4 \%$ fueron amenazadas con algún tipo de arma.

Las mujeres que vivían con la pareja abusiva, que no estaban casadas con esa pareja, representaron el porcentaje más alto de las participantes $(47.7 \%)$. La segunda incidencia más alta fue representada por las mujeres casadas que vivían con su abusador $(23.5 \%)$. Las mujeres participantes también reportaron ser abusadas por algún conocido cercano $(0.9 \%)$, por su conyugue de hecho $(0.7 \%)$, pareja de citas $(1.9 \%)$, y $1.7 \%$ reportaron haber sido abusadas por su conyugue, del cual estaban divorciadas. Las participantes también reportaron haber sido abusadas por su ex-pareja (5.2\%), exconyugue $(0.6 \%)$, y su pareja íntima con quien vivían (1.2\%). De todas las participantes, $3.2 \%$ reportaron haber sido abusadas por una pareja íntima con quien no estaban casadas y con quien no vivían; $0.2 \%$ reportaron haber sido abusadas por su amante. Las parejas intimas del mismo sexo y quienes vivían juntas representaron $0.3 \%$ de los abusadores; solo un caso de abuso fue registrado como abuso entre pareja intima del mismo sexo y quienes no vivían juntas. Las mujeres participantes también reportaron estar separadas de su abusador (6.8\%), estar separadas de de su conyugue abusivo $(0.6 \%)$ y $5.5 \%$ estaban siendo abusadas por su conyugue. Casi todas las participantes (90.8\%) en este estudio estuvieron en el albergue 90-dias o menos durante los años 2010 al 2015. El restante $9.2 \%$ permaneció en el albergue más de 90 días. 
Se observó un nivel muy bajo de participación en los módulos educativos ofrecidos en el albergue. La participación en el Programa Abriendo la Cerradura de Casa fue solamente de un 2.7\%. En el Programa de Empoderamiento Económico solamente participaron $24.4 \%$ de las mujeres residentes del albergue. El Programa de Preparación para Empleo únicamente registró 10.4\% de participación; este programa incluyó actividades relacionadas con la búsqueda de empleo, tales como ferias de trabajo, sesiones básicas de computación, y laboratorios de computadoras.

La prueba McNemar fue aplicada para analizar los datos, los cuales revelaron que la decisión tomada con respecto al alojamiento, según reportada por las mujeres abusadas que participaron en las sesiones educativas de alojamiento seguro, cambió significativamente $(p<.01)$. Las mujeres estaban más propensas a obtener alojamiento seguro lejos del su pareja abusiva cuando salían del albergue (58.4\%), en comparación con la información que éstas proveyeron a su llegada al albergue (45.5\%). Estos resultados indican que hubo una asociación positiva en términos del tema de alojamiento; por lo tanto, la hipótesis nula fue rechazada (Tabla 1).

Tabla 1

Alojamiento Seguro

\begin{tabular}{cccccc}
\hline & \multicolumn{2}{c}{$\begin{array}{c}\text { Alojamiento Seguro al } \\
\text { Ingresar }\end{array}$} & \multicolumn{2}{c}{ Alojamiento Seguro al Salir } & \\
\cline { 2 - 5 } Respuesta & Frecuencia & Porciento & Frecuencia & Porciento & $p$ \\
\hline Si & 35 & 45,5 & 45 & 58,4 & 0,002 \\
No & 42 & 54,5 & 32 & 41,6 &
\end{tabular}

Nota: Resultados de prueba McNemar respecto al alojamiento de las mujeres maltratadas participantes en el programa educativo Abriendo la Cerradura de Casa. Fuente: Propia (2017).

Otra de las variables incluidas en el análisis del tema de alojamiento fue el desarrollo completo de un plan de contingencia. Esta variable alude a un plan estratégico diseñado para obtener la seguridad de la víctima de violencia entre pareja intima, lejos de la pareja abusiva. Este plan de contingencia es un plan práctico y personalizado, el cual incluye acciones seguras para escapar en caso de alguna emergencia relacionada a la violencia entre pareja íntima, aun cuando esto implique regresar al albergue. Los resultados de la prueba McNemar resumidos en la Tabla 2, reflejan que todas las mujeres maltratadas de las cuales hubo información disponible $(n=63)$ y quienes participaron en el Programa Abriendo la Cerradura de Casa fueron empoderadas con un plan de contingencia. Esta es una diferencia significativa $(p<$ $.001)$, en comparación con los datos reportados al momento de ingresar al albergue $(27 \%)$. 
Tabla 2

Plan de Contingencia.

\begin{tabular}{cccccc}
\hline & \multicolumn{2}{c}{$\begin{array}{c}\text { Plan de Contingencia al } \\
\text { Ingresar }\end{array}$} & \multicolumn{2}{c}{ Plan de Contingencia al } \\
Respuesta & Frecuencia & Porciento & Frecuencia & Porciento & \multirow{2}{*}{$p$} \\
\cline { 2 - 5 } $\mathrm{Si}$ & 17 & 27,0 & 63 & 100,0 & $<0,001$ \\
$\mathrm{No}$ & 46 & 73,0 & 0 & 0 & \\
\hline
\end{tabular}

Nota: Resultados de la prueba McNemar sobre la finalización del plan de contingencia de las mujeres maltratadas participantes en el programa educativo Abriendo la Cerradura de Casa. Fuente: Propia (2017).

La prueba McNemar fue realizada para determinar si las mujeres maltratadas que participaron en el Programa de Empoderamiento Económico fueron empoderadas para obtener los recursos económicos que les permitieran escapar de la violencia entre pareja íntima. Debido a que todas las mujeres pertenecían a la categoría de "Ingreso igual o menos de $\$ 75,000$ ", la variable de ingreso económico (al entrar al albergue) fue mejor representada por su estado laboral a su llegada al albergue. Los datos de los planes para obtener ingreso económico fue re-codificado en una variable dicótoma y etiquetada como "plan de Ingreso" (a la salida del albergue).

Según muestra la Tabla 3, hubo una diferencia significativa en términos de problemas financieros $(p<.001)$; sin embargo, no se registraron grandes logros. Este resultado implica que las mujeres maltratadas que participaron en sesiones educativas relacionadas con el manejo financiero no se sienten empoderadas con destrezas ni recursos económicos. No se evidenció una asociación positiva, por lo tanto, se retuvo la hipótesis nula.

Tabla 3

Plan de Ingreso Económico

\begin{tabular}{cccccc}
\hline & \multicolumn{2}{c}{$\begin{array}{c}\text { Plan de Ingreso Económico } \\
\text { al Ingresar }\end{array}$} & \multicolumn{2}{c}{$\begin{array}{c}\text { Plan de Ingreso Económico } \\
\text { al Salir }\end{array}$} & \\
\cline { 2 - 5 } Respuesta & Frecuencia & Porciento & Frecuencia & Porciento & $p$ \\
\hline $\mathrm{Si}$ & 141 & 20,4 & 55 & 7,9 & $<0,001$ \\
$\mathrm{No}$ & 551 & 79,6 & 637 & 92,1 & \\
\hline
\end{tabular}

Nota: Resultados de la prueba McNemar sobre el empoderamiento para manejar finanzas de las mujeres maltratadas, participantes del programa educativo Empoderamiento Económico. Fuente: Propia (2017).

Para obtener una visión más profunda a través de este análisis de datos, se llevó a cabo una tabulación cruzada, comparando los planes para obtener ingreso de las mujeres que participaron en el programa educativo Empoderamiento Económico con aquellas que no participaron en éste. Un porcentaje ligeramente más alto de mujeres que participaron en estas sesiones educativas tuvieron planes para obtener ingreso económico, lo cual les permitiría lograr su independencia económica (7.9\%), comparadas con las mujeres que no participaron en este mismo programa (6.8\%). Estos resultados no representaban una diferencia estadística significativa $(p>.01)$ (Tabla 4). 
Tabla 4

Empoderamiento Económico

\begin{tabular}{|c|c|c|c|c|c|}
\hline \multirow[b]{2}{*}{ Respuesta } & \multicolumn{2}{|c|}{$\begin{array}{c}\text { No-participante del } \\
\text { Programa Empoderamiento } \\
\text { Económico con plan de } \\
\text { Ingreso Económico }\end{array}$} & \multicolumn{2}{|c|}{$\begin{array}{c}\text { Participante del Programa } \\
\text { Empoderamiento } \\
\text { Económico con plan de } \\
\text { Ingreso Económico }\end{array}$} & \multirow[b]{2}{*}{$p$} \\
\hline & Frecuencia & Porciento & Frecuencia & Porciento & \\
\hline $\mathrm{Si}$ & 145 & 6,8 & 55 & 7,9 & 0,288 \\
\hline No & 2000 & 93,2 & 637 & 92,1 & \\
\hline
\end{tabular}

Nota: Muestra de mujeres maltratadas clasificada de acuerdo a sus planes para obtener ingreso y su participación en el programa educativo Empoderamiento Económico. Fuente: Propia (2017).

La participación en el programa educativo Preparación para el Trabajo incluyó diferentes actividades tales como ferias de trabajo, sesiones básicas de computación, y laboratorios de computadora. La prueba McNemar fue utilizada para examinar los datos disponibles referentes al empleo de las participantes. La variable utilizada para representar el empleo de las participantes fue representada por su estado laboral al momento de su llegada al albergue. Por otra parte, la variable de empleo, al salir del albergue, fue construida por una re-codificación dicótoma de los planes de estas mujeres para obtener un ingreso económico, para lo cual se consideró los planes de unirse a las fuerzas laborales y a los cual se le agregaron los datos referentes a los planes para obtener empleo. El tomar en consideración ambas variables permitió obtener una mejor imagen de los planes de las mujeres maltratadas sobre sus planes de empleo a su salida del albergue.

Los resultados de la prueba McNemar, según se muestran en la Tabla 5, revelaron que no hubo una diferencia estadística significativa en proporción al estado de empleo al momento de su llegada al albergue y los planes de empleo al momento de su salida del albergue, después de haber participado en el programa educativo Preparación para el Trabajo $(p>.01)$. Se evidenció una incidencia de logro de empleo en muy pocas mujeres $(12.9 \%)$. Tomando en cuenta estos resultados, se retuvo la hipótesis nula. Los resultados sugieren que las mujeres maltratadas que tienen acceso a los programas educativos que las empoderan con las destrezas y los recursos para obtener empleo no está asociado con el hecho de unirse a la fuerza laboral lejos de una relación violenta entre pareja íntima.

Tabla 5

Preparación para el Trabajo

\begin{tabular}{cccccc}
\hline & \multicolumn{2}{c}{ Plan de Empleo al Ingresar } & Plan de Empleo al Ingresar & \\
\cline { 2 - 5 } Respuesta & Frecuencia & Porciento & Frecuencia & Porciento & $p$ \\
\hline Si & 52 & 17,7 & 38 & 12,9 & 0,110 \\
No & 242 & 82,3 & 256 & 87,1 & \\
& & & & &
\end{tabular}

Nota: Resultados de prueba McNemar sobre logros de empleo de mujeres maltratadas, participantes en el programa Preparación para el Trabajo. Fuente: Propia (2017).

Adicionalmente, se preparó una tabulación cruzada para comparar los planes de empleo de las mujeres maltratadas que participaron en el programa Preparación para el 
Trabajo y los planes de empleo de aquellas mujeres que no tuvieron esta experiencia educativa. Los datos en la Tabla 6 demuestran que un porcentaje más alto de las mujeres que participaron en el módulo educativo tenían planes de empleo que les permitirían unirse a las fuerzas laborales lejos de su relación violenta entre pareja intima (13.1\%), comparado con el de las mujeres que no participaron en la sesión educativa $(6.9 \%)$. Estos resultados representan una diferencia estadística significativa $(\mathrm{p}<.001)$.

Tabla 6

Planes de Empleo y Preparación para el Trabajo

\begin{tabular}{cccccc}
\hline & $\begin{array}{c}\text { No-participante del Programa } \\
\text { Preparación para el Trabajo } \\
\text { con plan de Empleo }\end{array}$ & $\begin{array}{c}\text { Participante del Programa } \\
\text { Preparación para el Trabajo } \\
\text { con plan de Empleo }\end{array}$ & & \\
\cline { 2 - 5 } Respuesta & Frecuencia & Porciento & Frecuencia & Porciento & $p$ \\
\hline Si & 175 & 6,9 & 39 & 13,1 & $<0,001$ \\
No & 2365 & 93,1 & 258 & 86,9 & \\
& & & & & \\
& & & & &
\end{tabular}

Nota: Muestra de las mujeres maltratadas, con planes de empleo y participación en el Programa Preparación para el Trabajo. Fuente: Propia (2017).

\section{Discusión y conclusiones}

Aún cuando el enfoque de este estudio fue el empoderamiento de mujeres maltratadas, menos del $25 \%$ de las mujeres residentes del albergue participaron en los programas educativos ofrecidos en este. Estos resultados reflejan el uso limitado de los servicios disponibles en el albergue de mujeres maltratadas e implica que las mujeres maltratadas desconocen la importancia de la educación como una herramienta de empoderamiento para obtener mejores oportunidades para separarse de su pareja abusiva.

El hecho de que las mujeres que participaron en el programa educativo Abriendo la Cerradura de Casa fueron más propensas a obtener alojamiento seguro lejos lejos de su pareja abusiva cuando salían del albergue, implica que los programas educativos que empoderan a las mujeres maltratadas con las destrezas y los recursos para obtener alojamiento seguro son significativamente efectivos para ayudarlas a salir de una relación entre pareja íntima violenta. Aun cuando los resultados revelaron que la gran mayoría de las mujeres que participaron en este programa educativo fueron empoderadas con un plan de contingencia diseñado para lograr mayor seguridad y su libertad lejos de su pareja abusiva, es necesario implementar estrategias prácticas y objetivas para evaluar la efectividad de este plan de contingencia una vez abandonan el albergue de mujeres maltratadas.

Los resultados del análisis estadístico de la participación en el Programa Empoderamiento Económico reveló que solo un pequeño porcentaje de las mujeres que participaron en este programa educativo reportaron tener planes que les permitirían obtener independencia financiera, sugiriendo que las mujeres maltratadas que tuvieron acceso a este programa educativo que las empodero con las destrezas y los recursos para manejar sus finanzas no aumentó su ingreso para ayudarlas a mantenerse alejadas de su pareja íntima abusiva. Estos resultados implican que existe la necesidad de investigar más a fondo la efectividad de programas que ayudan a la mujer a conseguir su 
independencia económica y así disminuir su necesidad de regresar con su pareja abusiva.

Varios programas de empoderamiento económico que han sido implementados a escala nacional han reportado una alta tasa de éxito en el empoderamiento económico de mujeres maltratadas; por lo tanto, los resultados de este análisis sugieren que aún existe la necesidad de utilizar programas eficaces como modelos para diseñar e implementar programas de empoderamiento económico que se adapten a las numerosas circunstancias que afectan a las mujeres víctimas de violencia entre pareja íntima.

El análisis estadístico de los expedientes de las mujeres que participaron en el módulo educativo Preparación para el Trabajo reveló que estas participantes no experimentaron un cambio significativo en términos de sus planes de empleo; por lo tanto, se implica que las mujeres abusadas que tienen acceso a programas educativos que las empoderan con las destrezas y los recursos para obtener empleo no necesariamente lo obtienen. Esta incidencia refleja la necesidad de investigar más a fondo los factores que afectan la incidencia de búsqueda de empleo en este tipo de población. El hecho de que la tabulación cruzada reveló que un alto porcentaje de las participantes en este módulo educativo reportaron tener planes de empleo, sugiere una necesidad de poder dar seguimiento y evaluar si los planes reportados se llevaron a cabo una vez la victima abandona el albergue.

Una de las implicaciones más importantes de este estudio es el hecho de que los diferentes programas que se ofrecen en los albergues para mujeres maltratadas proveen, con ciertas limitaciones, resultados positivos. Los resultados varían según cada programa, así como también varían las necesidades de cada víctima; más aún, este análisis retroactivo también reitera lo que otros estudios han evidenciado: los programas de asistencia para víctimas de violencia entre pareja íntima proveen servicios a un sinnúmero de mujeres que sufren las consecuencias de la violencia a manos de una pareja intima abusiva, las cuales van más allá de la violencia física.

Los efectos observados en este análisis de la participación de mujeres en programas educativos de empoderamiento, aunque relativamente pequeños, fueron previstos debido a la complejidad de los problemas que rodean la violencia entre pareja íntima, así como la amplia variedad de disponibilidad y acceso de servicios dentro y fuera de los albergues y agencias de apoyo para mujeres maltratadas. Es poco probable que los efectos de empoderamiento de las víctimas de violencia entre pareja íntima a través de servicios educativos puedan ser evaluados con precisión sin los recursos adecuados que representen la medición de los logros obtenidos.

Otra implicación importante de este estudio es la necesidad de utilizar programas educativos basados en evidencia científica con componentes principales efectivos para educar a las víctimas de violencia entre pareja íntima y cambiar su mentalidad sobre sus necesidades y promover así una calidad de vida más segura y satisfactoria. Aún cuando este análisis se enfocó únicamente en programas educativos que abordaban el tema de los problemas de alojamiento, ingreso económico, y empleo, con el fin de analizar el impacto de empoderamiento de las mujeres sobrevivientes de violencia entre pareja intima, se necesita más evidencia para apoyar la especulación de que la participación en programas educativos ofrecidos en los albergues de mujeres maltratadas empodera a las mujeres víctimas de violencia entre pareja intima a ser participantes activas y en control de su recuperación de su recuperación.

No todos los programas educativos que apoyan a las víctimas de violencia entre pareja íntima tienen como propósito principal proveer herramientas de emancipación 
para este tipo de víctimas. Estos programas no siempre tienen como objetivo primordial proveer a las mujeres victimizadas las estrategias de empoderamiento necesarias para resolver sus necesidades de alojamiento, empleo y solvencia económica, lo cual genera otros problemas de salud emocional. Adicionalmente, otra de las implicaciones relacionadas a esta falta de objetivos estandarizados es que mientras las agencias de ayuda para mujeres maltratadas no consideren el empoderamiento de las victimas una prioridad, estas se abstendrán de mejorar los servicios educativos disponibles actualmente y sus intervenciones continuarán sin el propósito de crear cambios positivos.

Mientras no todas las residentes del albergue de mujeres maltratadas participen en los servicios educativos ofrecidos, sería de gran beneficio evaluar las razones por las cuales la víctima se abstiene de utilizar estos servicios; sin embargo, los esfuerzos educativos deberían continuar, ya sea a través de los servicios ofrecidos directamente en el albergue o a través de la colaboración de agencias de la comunidad; sin embargo, como muchos de los problemas relacionados con estos servicios se extienden más allá de la capacidad y jurisdicción de cada institución, los programas educativos deberían incluir redes de recursos disponibles para incorporar el apoyo social y el acceso a otras agencias comunitarias y gubernamentales que contribuyan al éxito de las intervenciones implementadas. Aun cuando los resultados a largo plazo no pudieron ser evaluados en este estudio, los resultados inmediatos evidenciaron la necesidad de dar seguimiento a las víctimas de violencia entre pareja intima una vez abandonan el albergue.

La mayoría de los estudios actuales no interpretan las diferencias fundamentales entre las participantes que reciben servicios en al albergue de mujeres maltratadas y aquellas que no los reciben. Esta limitación implica que cualquier resultado atribuido a la utilización de servicios no es necesariamente debido a la efectividad de la implementación del servicio ofrecido, si no a alguna característica específica que tienen en común las victimas que participan en este tipo de programas. Cuando los programas son evaluados utilizando métodos que permiten parcialidad en su selección y no incluyen la interpretación de todos los hallazgos, se puede especular erróneamente que los programas no son efectivos. El poder determinar el por qué estos programas educativos no registran una alta tasa de éxito entre las mujeres víctimas de violencia entre pareja intima es el primer paso hacia el desarrollo de recursos efectivos que empoderan a la mujer para escapar una relación violenta.

En conclusión, los hallazgos de este análisis sugieren que solo pocas mujeres participan en programas de empoderamiento ofrecidos en los albergues de mujeres maltratadas. Adicionalmente, los resultados de este estudio retrospectivo se añaden a las estadísticas que reportan que las mujeres víctimas de violencia entre pareja íntima buscan más la asistencia informal que los recursos formales de apoyo. Estos hallazgos continúan revelando datos significativos sobre el impacto de violencia entre pareja intima en contra de la mujer y la falta de intervenciones efectivas que las empoderen para romper el ciclo de violencia que controla su vida.

Las estrategias que afrontan los problemas relacionados a la violencia entre pareja íntima y la educación, empleo, alojamiento, problemas económicos, legalidades y parcialidad de género, entre otras, juegan un papel central en la evolución de soluciones efectivas que abordan la violencia entre pareja intima en contra de la mujer.

Los esfuerzos de investigación deberían continuar con el fin de estudiar la efectividad de programas educativos de empoderamiento ya que se necesita evidencia adicional para apoyar la presunción de que la participación en programas educativos 
ofrecidos en albergues de mujeres maltratadas empodera a las víctimas de violencia entre pareja íntima para tomar control de su vida alejada del ciclo de violencia.

\section{Referencias}

Aiken, J., \& Goldwasser, K. (2010). The perils of empowerment. Cornell Journal of Law and Public Policy, 20(139). Recuperado de http://scholarship.law.georgetown.edu/cgi/viewcontent.cgi?article=1500\&contex $\mathrm{t}=$ facpub

Asian Pacific Institute on Gender-Based Violence. (2015). Patriarchy and power. Recuperado de http://www.api-gbv.org/violence/patriarchy-power.php

Criminal Justice. (n.d.). Worldwide history of domestic violence. Recuperado de http://criminal-justice.iresearchnet.com/crime/domestic-violence/worldwidehistory-of-domestic-violence/

Dare, B., Guadagno, R., \& Muscanell, N. (2013). Commitment: the key to women staying in abusive relationships. Journal of Interpersonal Relations, 6. Recuperado http://onlinesocialinfluence.net/.pubs/DareGuadagnoMuscanell_2013.pdf

Erez, E. (2002). Domestic violence and the criminal justice system: an overview. The Online Journal of Issues in Nursing, 7. Recuperado de http://www.nursingworld.org/MainMenuCategories/ANAMarketplace/ANAPeri odicals/OJIN/TableofContents/Volume72002/No1Jan2002/DomesticViolencean dCriminalJustice.html

Kreager, D., Felson, R., Warner, C., \& Wenger, M. (2013). Women's education, marital violence and divorce: A social exchange perspective. Journal of Marriage and Family, 75. Recuperado de https://www.ncbi.nlm.nih.gov/pmc/articles/PMC3864686/

Missouri Coalition Against Domestic and Sexual Violence. (2014). The nature and dynamics of domestic violence. Recuperado de http://www.mocadsv.org//FileStream.aspx?FileID=74

Ofstehage, A., Gandhi, A., Sholk, J., Radday, A., \& Stanzler, C. (2011). Empowering victims of domestic violence. Recuperado de http://www.rootcause.org/docs/Resources/Research/Empowering-Victims-ofDomestic-Violence/Empowering\%20Victims\%20of\%20Domestic\%20Violence$\%$ 20Social\%20Issue\%20Report.pdf

Temple, J., Le, V., Muir, A., Goforth, L., \& McElhany, A. (2013). The need for school based teen dating violence prevention. Journal of Applied Research on Children: Informing Policy for Children at Risk, 4. Recuperado de http://digitalcommons.library.tmc.edu/childrenatrisk/vol4/iss1/4

Texas Council on Family Violence. (2014). Texas family violence facts and statistics. Recuperado de http://www.tcfv.org/pdf/Texas_Family_Violence_Facts_and_Statistics.pdf

Vaile-Wright, C., Perez, S., \& Johnson, D. (2012). The attenuating effect of empowerment on IPV-related PTSD symptoms in battered women living in 
domestic violence shelters. Violence Against Women, 18. Recuperado de http://www.ncbi.nlm.nih.gov/pmc/articles/PMC3305196/

Wihbey, J. (2015). Domestic violence and abusive relationships: Research review. Recuperado de https://journalistsresource.org/studies/society/gendersociety/domestic-violence-abusive-relationships-research-review

World Health Organization. (2009). Promoting gender equality to prevent violence against women. Recuperado de http://www.who.int/violence_injury_prevention/violence/gender.pdf

Fecha de recepción: 16/06/2017

Fecha de revisión: 03/10/2017

Fecha de aceptación: 16/11/2017 